\title{
The EHE Neutrino Search Capability of the IceCube Observatory
}

\author{
Aya Ishihara ${ }^{a *}$ for the IceCube Collaboration

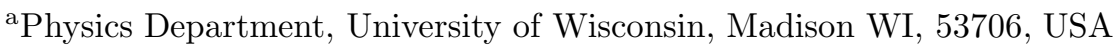

An initial study of the extremely high energy (EHE) physics capability of the IceCube neutrino observatory is demonstrated by considering a GZK mechanism neutrino production model, which is a guaranteed source for EHE neutrinos. We study EHE event properties in the energy range $10^{5}<\mathrm{E}<10^{11} \mathrm{GeV}$ observed by IceCube using detailed Monte Carlo simulation. Results of a simulation study show that about 0.7 EHE neutrino events per year is expected with the full IceCube configuration over a 0.03 atmospheric muon background which passed an EHE event criteria. It is also shown that with the present partial IceCube detector with the same criteria is capable of studying EHE physics and the sensitivity improves with the number of deployed strings in the ice.

\section{Introduction}

It is well known that there exists extremely high energy (EHE) particles in the universe with energies approaching up to $\sim 10^{20} \mathrm{eV}$ [1]. These EHE cosmic rays (EHECRs) may produce neutrinos by various mechanisms, namely when they interact with surrounding matter or photon fields. The neutrinos are generated by the decays of $\pi$ mesons via $\pi^{ \pm} \rightarrow \mu^{ \pm} \nu_{\mu} \rightarrow e^{ \pm} \nu_{e} \nu_{\mu}$ process. In the EHE region, collisions of EHECRs and the cosmic microwave background photons produce cosmogenic neutrinos [2], a consequence of a process known as the Greisen-Zatsepin-Kuzmin (GZK) mechanism [3].

In general neutrinos are unique probes for exploring the high energy universe mainly because of two distinct features. The first feature is that the existence of cosmic neutrinos implies that there are hadron beams because only high energy hadronic processes can produce neutrinos. The second feature is that neutrinos can penetrate dense matter and radiation fields because of their small cross-sections, and therefore can propagate over cosmological distances. This implies that by observing neutrinos, one is able to learn about stars and galaxies which are surrounded by dense gases or energetic objects at very large distances. Such information is obscured when ob-

*Present address: Dept. of Physics, Chiba University, Chiba 263-8522 Japan serving with photons (e.g., radio, visible light, Xray and $\gamma$-ray). Because of these features, furthermore, detection of EHE neutrinos may shed light onto one of the most difficult questions in modern astrophysics, how and where are EHECRs produced.

It is generally very difficult to detect high energy neutrinos because one needs a large target volume for neutrinos to interact and produce a detectable signal in a reasonable time scale. The IceCube neutrino observatory, however, provides a rare opportunity to overcome this difficulty. Its $\mathrm{km}^{3}$ fiducial volume which uses clean glacier ice deep below the surface at the South Pole is a powerful tool to search for EHE neutrinos. In this paper we discuss the capability of the IceCube experiment to detect EHE neutrinos.

The paper is outlined as follows: In the next section, the overview of the IceCube observatory and its present status are briefly mentioned. The section 3 describes the characteristics of EHE neutrino events in the IceCube detector. We explain signals and backgrounds in our EHE neutrino search and a possible strategy to discriminate neutrino events by considering their event topologies. In the section 4 , the main results from the detailed Monte Carlo simulation study are shown, referring to the preliminary numbers concerning effective area and event rate for cosmogenic EHE neutrinos. We summarize our conclusion and future prospects at the end. 


\section{The IceCube Neutrino Observatory}

IceCube is a next-generation cubic-kilometer scale high energy cosmic neutrino telescope currently under construction and in operation at the geographic South Pole. It uses $3 \mathrm{~km}$ thick glacial ice as a Cherenkov medium. Cherenkov photons emitted from relativistic charged particles such as muons are received by an array of Digital Optical Modules (DOMs) which amplify and digitally sample the high-speed photomultiplier tube (PMT) pulses in situ. Each DOM encloses 10" R7081-02 PMT made by Hamamatsu Photonics in a transparent pressure sphere along with the high voltage system, a LED flasher board for optical calibration in ice, and a digital readout board. The deep-ice DOMs are deployed along electrical cable bundles which carry power and information between the DOMs and surface electronics. The cable assemblies, often called strings, are dropped into holes drilled to a depth of 2450 meters. The DOMs occupy the last 1000 meters at intervals of 17 meters where the glacial ice is transparent. DOMs are also frozen into tanks located at the surface near the top of each hole which constitutes an air shower array called IceTop. IceTop provides us with the capability to study the atmospheric muon background reliably. This feature plays a key role in the IceCube EHE neutrino search as we will mention later. The strings and tanks are arranged in a hexagonal lattice pattern with a spacing of approximately 125 meters. At completion (planned to be 2011), the array will comprise 4200 in-ice DOMs on 70 strings and 320 modules in the surface array. Currently, IceCube includes 9 in-ice strings (540 DOMs) and 32 IceTop tanks (68 DOMs).

Events recorded by IceCube are generally categorized by geometrical patterns of photon distributions from the minimum ionizing in-ice charged particles. The "track" events are initiated either from penetrating neutrino-induced muons or taus, or from muons coming from extensive air shower cascades above the surface i.e., the atmospheric muons. Directional reconstruction of these tracks suppresses the atmospheric muon background. By selecting neutrino events that are up going, this effectively eliminates the at- mospheric muon background since they are down going. The other event pattern known as a "cascade" is induced by an electromagnetic (EM) cascade via the $\nu_{e}$ charged-current interaction or hadronic cascades via the neutral-current interaction of all neutrino flavors. Those cascades generated inside IceCube detection volume emit spherical shower-like patterns of Cherenkov light. Because muon and tau tracks have a longer propagation length in matter/ice than that of the electron and hadron cascades, tracks generally have a larger effective area.

EHE events that are expected to be seen by the IceCube observatory can be considered beloinging to neither class, however, because of difference in their major energy-loss processes. At the highest energies, photon emission patterns differ and their remarkably sizable energy deposit in the IceCube array may create a different characteristic signature. We describe this EHE event topology in the next section.

\section{EHE neutrino events in IceCube}

In the EHE region, because of the increase of the neutrino cross-section with energy, neutrinos are more likely to be involved in interactions with matter during their propagation than to penetrate through the Earth. Charged leptons and hadrons are generated in these interactions and the secondary produced $\mu$ 's and $\tau$ 's travel the Earth losing their energies by undergoing many radiative reactions, i.e. EM cascades generated by $e^{ \pm}$pair creation, Bremsstrahlung, and hadronic cascades generated by the photonuclear interactions. The IceCube detector is to observe these secondary $\mu$ 's and $\tau$ 's as a main detectable channel of EHE signals [4]. There are two prominent characteristics for these EHE neutrino events. One is that a major fraction of them arrive at the detector with a down-going geometry because the mean free path of EHE neutrinos is much shorter than the typical path length in the Earth. Another is that the muon/tau "track" is accompanied with many "cascades" originating from the various radiative energy loss processes. The first characteristic makes it difficult to discriminate between the atmospheric muon 
background events and neutrino induced events by using the Earth as a filter. The second point, however, provides another way to distinguish the EHE signals from background because the energy loss due to stochastic radiative processes is proportional to the energy of muon(tau).

A measurement of the energy deposit inside the IceCube detection volume, therefore, leads to an estimation of the track energy. The estimated track energy tells if it is of cosmic origin. This is because the expected spectra of secondary $\mu$ 's and $\tau$ 's generated from the GZK neutrinos is much harder than that of atmospheric muons [4], and the measured energy (or its indicator) should be able to exclude the atmospheric muon events in a relatively straightforward manner. Figure 1 shows examples of simulated IceCube events at different energies. One can see that the EHE muon radiates a large number of Cherenkov photons. The amount of Cherenkov photons in an event, the "brightness" of the event in other words, appears to indicate the track energy. Detailed Monte Carlo study has shown that the number of detected photons is a robust energy indicator to search for EHE signals, which is described in the following section.

\section{Results}

\subsection{Simulation Setup and Signal Domain Criteria}

Transportation of the EHE neutrinos and their secondary particles from the Earth's surface to the detector depth is calculated by the JULIeT package [5] as described in Ref. [4]. The IceCube Monte Carlo simulation package then generates events with energies and intensities following the obtained fluxes. The primary cosmic neutrino flux in this paper is assumed to be GZK cosmogenic neutrinos as calculated in Ref. [6]. For the atmospheric muon flux, which is considered our main background, we take the analytically fitted result using a Corsika simulation [7] assuming an initial cosmic-ray proton flux of the form of $E^{-3}$. We should note that the atmospheric muon background in the EHE regime is highly uncertain due to our poor knowledge of the EHECR mass composition and the muon bundle inten-

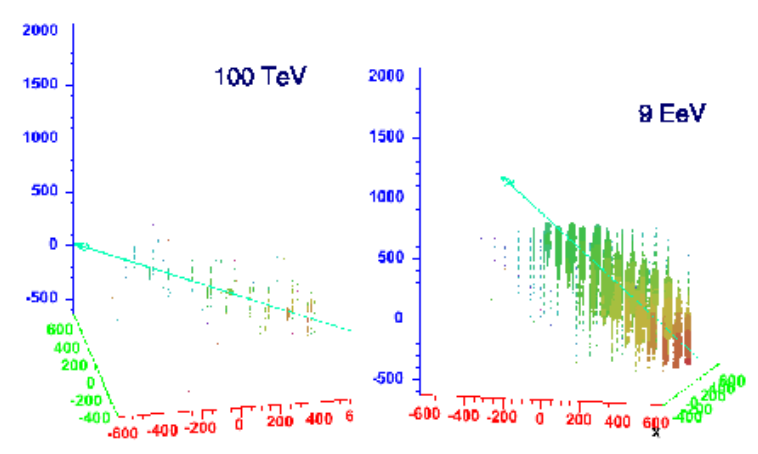

Figure 1. Examples of simulated IceCube events. Left panel shows a $100 \mathrm{TeV}$ muon track representing the conventional IceCube event while the right panel indicates a $9 \mathrm{EeV}$ muon EHE event. Colored circles denote DOMs with more than one photon, the size of the circles represents the number of photons and the color indicate the first photon arrival time at each DOMs. $\mathrm{x}, \mathrm{y}$ and $\mathrm{z}$ axes are distances from the central position of the IceCube detector.

sity. The background intensities will be deduced from the IceCube data in the future. The present simulation chain considers events induced by the charged leptons that interact within $860 \mathrm{~m}$ from the center of detector because the secondary produced muons and taus are our main EHE events in IceCube [4]. The energy range under consideration is between $10^{5}$ and $10^{11} \mathrm{GeV}$ near the detector.

As described in Section 3, the total amount of Cherenkov light detected for an event is expected to be sensitive to the energy of incoming EHE muon or tau. The IceCube detector can measure the Cherenkov luminosity per event as the event-total number of photo-electrons (NPE) detected by each DOM. Figure 2 shows distributions of NPE as a function of the muon and tau energy. It shows a correlation between NPE and the muon/tau energy going through the IceCube volume. Notice that the track geometry also affects the observed NPEs since a track passing further 


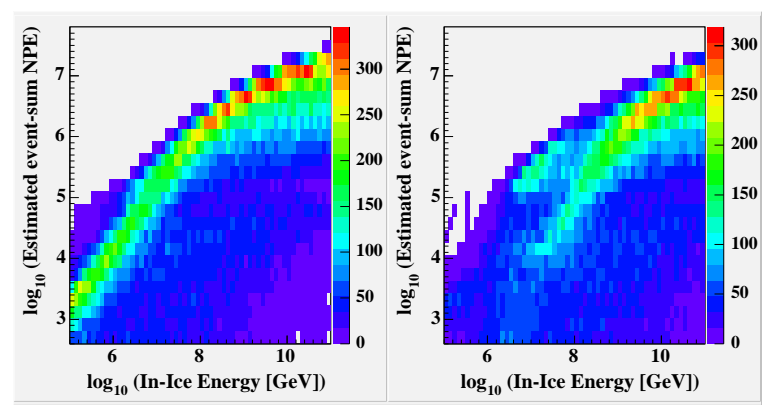

Figure 2. Simulated event distributions on a plane of NPE and the charged lepton energy. Energy plotted here is the value when the incoming particle is at the surface of IceCube detection volume which is defined as a sphere of 860 meters in radius in the present study. The left plot shows muon events and the right plot shows tau events. A suppression of energy loss for taus compared to that of muons and the contributions from taudecays are visible in the right plot.

away from the DOMs will result in less Cherenkov light within the IceCube detector. A distinct feature in the plots is that the primary energy of the incoming leptons is still a deciding factor in the observed NPEs, which implies that NPE is a robust indicator of the lepton energy without having to rely on event geometry reconstruction.

The zenith angle distributions are quite different for muon and taus originating from the propagation of cosmogenic GZK neutrinos and atmospheric muons, however. As down-going GZK muons and taus have a higher tendency to arrive from near the horizontal direction than the atmospheric background [4], one can expect a difference in the distribution of zenith angles and NPE for EHE signals and background. Plotted in Figure 3 are the simulated event distributions on the plane of NPE and zenith angle of muons and taus expected from the GZK model and the atmospheric muon backgrounds, which behave consistently with our expectation. Taking advantage of such differences, we introduce the signal domain on the NPE-Zenith angle plane where the GZK

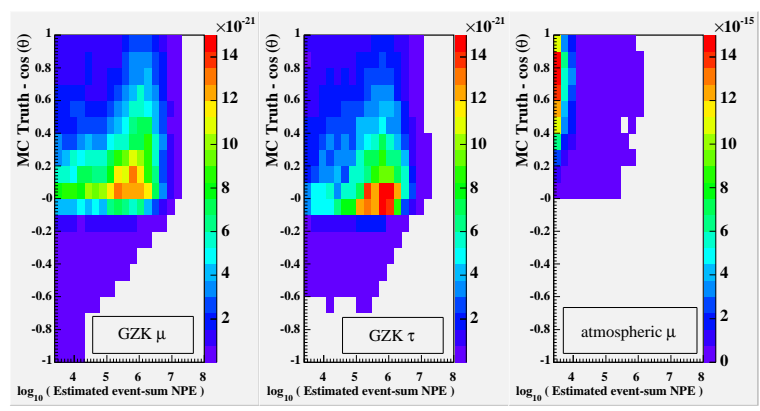

Figure 3. Event distribution on the plane of NPE and cosine of zenith angle obtained by Monte Carlo simulation. Plotted in the left and middle are that of GZK neutrino-induced muon and tau signals respectively and the background atmospheric muons on the right.

events dominates over the background defined as

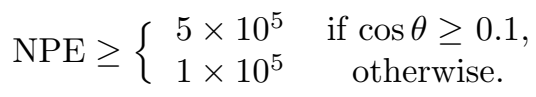

The expected event rate and effective area with this criteria are discussed next.

\subsection{Event Rate}

The expected signal and background event rate are shown in Figure 4 as functions of NPE and zenith angle.

Major parts of the dominant atmospheric background in the left plots are rejected by the selection criteria defined as Eq. (1) in the right plots. With these selection criterion, the GZK muon and tau signal events dominate. It is also found that the number of tau events are comparable to or even greater than that of muon events. In the EHE regime, the tau does not decay but is subject to radiative energy loss processes. Its heavier mass suppresses the energy loss compared to muons with the same energy, which gives a higher survival probability from near the horizontal direction.

Shown in Table 1 are the event rates from different string numbers deployed in ice. The same selection criteria are applied for all the string configurations, including the present 9 string setup. Event rate increases almost constantly as more strings are installed. 

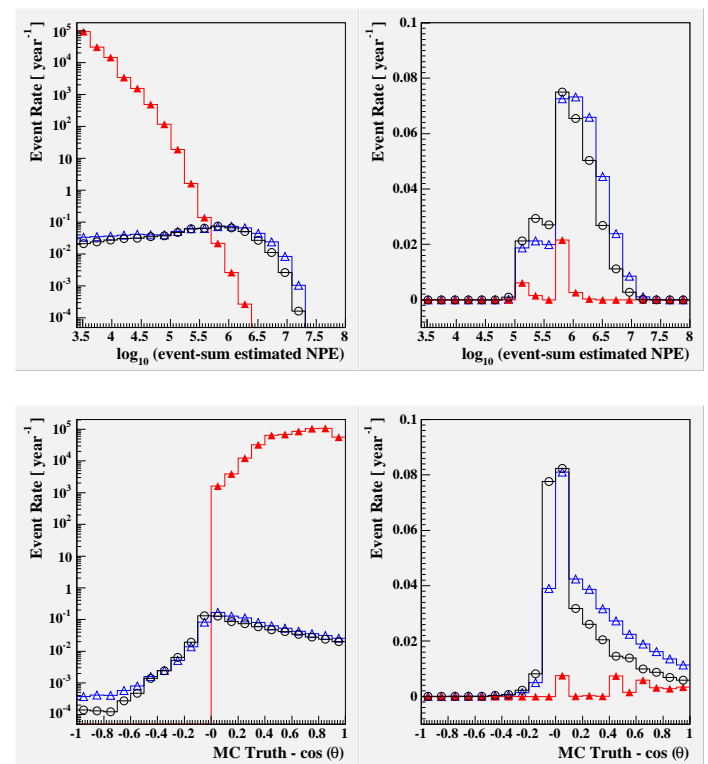

Figure 4. $\mu / \tau$ GZK signal and atmospheric $\mu$ event rate as function of NPE (upper) and $\cos (\theta)$ (lower). Blue and black markers indicate GZK $\mu, \tau$ respectively and red markers indicate atmospheric $\mu$. Left plots display event rates without background cut and right plots show after the signal domain selection. Multiple muon events are not included in the atmospheric muon flux.

\subsection{Effective Area}

The effective area resulted from the EHE signal domain cut is plotted in Figure 5 as a function of incoming charged lepton energy for different zenith angles. The NPE-based EHE event selection criteria decreases effective area in lower energy region while it enhances the detection efficiency for events with energies above $10^{8} \mathrm{GeV}$. These events are so luminous that the DOMs receive many photons even from the tracks propagating far outside the instrumented detector volume. The area reaches $\sim 2 \mathrm{~km}^{2}$, a factor of two larger than the IceCube physical cross section in energy region above $10^{10} \mathrm{GeV}$. The area for upgoing geometry is slightly larger as the NPE cut is loose for those geometries.
Table 1

The preliminary IceCube EHE event rate for different deployed in-ice string configurations. Muons and taus produced from the GZK model and the muon atmospheric background are shown.

\begin{tabular}{lccccc}
\hline string number & \multicolumn{1}{c}{9} & \multicolumn{1}{c}{20} & \multicolumn{1}{c}{40} & \multicolumn{1}{c}{60} & \multicolumn{1}{c}{80} \\
\hline GZK $\mu$ & 0.067 & 0.12 & 0.21 & 0.28 & 0.35 \\
GZK $\tau$ & 0.055 & 0.11 & 0.19 & 0.25 & 0.31 \\
atmospheric $\mu$ & 0.009 & 0.010 & 0.012 & 0.025 & 0.033 \\
\hline
\end{tabular}
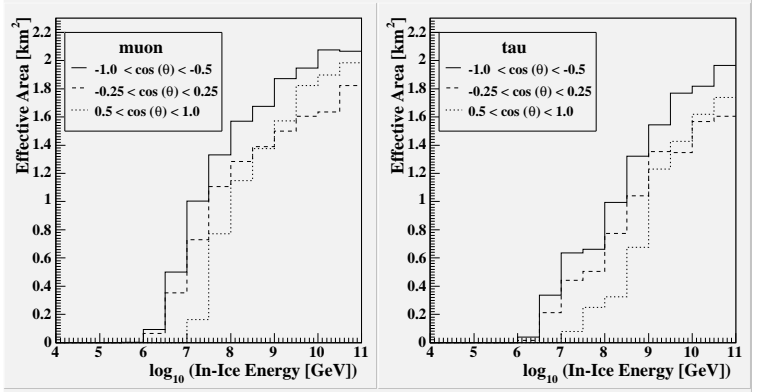

Figure 5. Muon (left) and tau (right) effective area as a function of incoming track energy with the 80 string configuration based on the EHE signal criteria. Dashed line denotes horizontal events and dotted line represents down-going events. Solid line indicates up-going events, although the probability of having EHE neutrinos with up-going geometry is very low, because of the Earth's sheltering effect.

\section{Summary and Future Prospective}

We have considered the capability of the IceCube detector in the search for EHE neutrinos without extensive energy reconstruction. The first level background rejection and signal selection is performed in terms of NPE and MC truth incoming direction. With this selection criteria, it is shown by a Monte Carlo simulation that about 0.7 EHE GZK events per year is expected with the full IceCube configuration over a background of 0.03 atmospheric muons.

The expected GZK event rate with the present 
9 string configuration is about 0.12 events per year with the same event selection. The number increases with the number of strings deployed from 9 strings to the full number of strings.

The study presented in this paper has not involved energy and geometry reconstruction of muon and tau tracks. We expect that good geometry reconstructions will support our results based on the Monte Carlo truth angle information. In addition, utilizing energy reconstruction should lead to a significant improvement of detector performance, especially for uncontained events that traverse outside the IceCube instrumentation volume. Ongoing development of EHE reconstruction methods will improve our sensitivity to EHE cosmic neutrinos in near future.

The results we have shown here heavily rely on NPE-Energy relations. These contain sizable uncertainties due to our incomplete understanding of the ice properties and detector responses to high NPE signals. The ice properties include the optical properties of the glacier ice and the behavior of the Cherenkov photon propagation in the hole ice which was melted and refrozen during the detector deployment. In order to reduce the systematic errors in the present study, the absolutely calibrated light source called the standard candle (SC) has been deployed in neighborhood of the DOMs in ice. The SC consists of a nitrogen laser, the calibrated PMT, and the related optics system and is able to emit pulses which are approximately equivalent to a $\mathrm{PeV} \mathrm{EM}$ cascade. Analyzing the data generated by the SC should reduce current uncertainties and improve the reliability of the EHE neutrino search.

The intensity of the atmospheric muon background in the EHE energy region is not well understood as we mentioned in Section 4, and we have assumed the Corsika-based analytical model in the present simulation study. In the future, however, it is expected that reasonable statistics of the data with NPE below $\sim 10^{5}$, allows us to build a reasonable empirical model to predict number of the background events in the EHE signal domain. We also would like to point out that in any case our sensitivity would not be changed significantly by varying the definition of energy based background rejection factor because the
GZK neutrino induced muons and taus exhibit an extremely hard energy distribution in contrast to the very soft $\left(\sim \mathrm{E}^{-4}\right)$ spectrum of the atmospheric muons. Besides, the atmospheric muon events can be experimentally identified and excluded by looking for coincidence events between the deep-ice IceCube strings and the IceTop surface array. With this power of the IceTop air shower array, the hybrid analysis using the inice DOMs and the surface DOMs, even with a limited data statistics, provides us further information about the background, including events consist of multiple muons, in the very high energy region.

\section{Acknowledgments}

We acknowledge the support from the following agencies: National Science Foundation-Office of Polar Program, National Science FoundationPhysics Division, University of Wisconsin Alumni Research Foundation, Department of Energy, and National Energy Research Scientific Computing Center (supported by the Office of Energy Research of the Department of Energy), the NSFsupported TeraGrid system at the San Diego Supercomputer Center (SDSC), and the National Center for Supercomputing Applications (NCSA); MEXT (Ministry of Education, Culture, Sports, Science, and Technology) in Japan; Swedish Research Council, Swedish Polar Research Secretariat, and Knut and Alice Wallenberg Foundation, Sweden; German Ministry for Education and Research, Deutsche Forschungsgemeinschaft (DFG), Germany; Fund for Scientific Research (FNRS-FWO), Flanders Institute to encourage scientific and technological research in industry (IWT), Belgian Federal Office for Scientific, Technical and Cultural affairs (OSTC); the Netherlands Organisation for Scientific Research (NWO); M. Ribordy acknowledges the support of the SNF (Switzerland); J. D. Zornoza acknowledges the Marie Curie OIF Program (contract 007921). 


\section{REFERENCES}

1. For a review see, e.g., M. Nagano and A. A. Watson, Rev. Mod. Phys. 72, (2000) 689; J. W. Cronin, Rev. Mod. Phys. 71, (1999) S165; S. Yoshida and H. Dai, J. Phys. G: Nucl. Part. Phys. 24, (1998) 905.

2. V. S. Beresinsky and G. T. Zatsepin, Phys. Lett. 28B, (1969) 423.

3. K. Greisen, Phys. Rev. Lett. 16, (1966) 748; G. T. Zatsepin and V. A. Kuzmin, Pisma Zh. Eksp. Teor. Fiz. 4, (1966) 114 [JETP. Lett. 4, (1966) 78].

4. S. Yoshida, R. Ishibashi, H. Miyamoto Phys. Rev. D 69 (2004) 103004.

5. http://www.ppl.phys.chiba-u.jp/JULIeT/

6. S. Yoshida and M. Teshima, Prog. Theor. Phys. 89, (1993) 833.

7. D. Heck et al., Report FZKA 6019, (Forschungszentrum Karlsruhe 1998). 